\title{
42. POROSITY AND VELOCITY VS. DEPTH AND EFFECTIVE STRESS IN CARBONATE SEDIMENTS ${ }^{1}$
}

\author{
Michael T. Hurley² and Peter Hempel ${ }^{3}$
}

\begin{abstract}
Results from a series of laboratory geotechnical and acoustical tests on carbonate sediments from the Western Indian Ocean are presented. With these data, empirical relationships for variations in porosity $(\phi)$ and compressional and shear wave velocity $\left(\mathrm{V}_{p}\right.$ and $\left.\mathrm{V}_{s}\right)$, with effective stress $\left(\sigma^{\prime}\right)$, were established. With an $\sigma^{\prime}$ vs. depth $(z)$ profile, nontemperature-corrected empirical equations for $\mathrm{V}_{p}$ and $\mathrm{V}_{s} \mathrm{vs}$. $z$ were then obtained. These data cover cover only the upper $100 \mathrm{~m}$ of sediments. An empirical equation for the variation of shear modulus (G) with $z$ is then established. This is shown to be dominated by the variation of $\mathrm{V}_{s}$ with $z$.

The importance of these equations in developing geoacoustical models of the seafloor, which accurately represent in-situ conditions, is assessed. A check of internal consistency was made: values of $\mathrm{V}_{p}$ were calculated with $\mathrm{V}_{s} \mathrm{vs}, \sigma^{\prime}$ and $\phi$ vs. $\sigma^{\prime}$ equations in Gassmann's theory, and comparisons are made with $\mathrm{V}_{p}$ vs. $\sigma^{\prime}$ empirical predictions. Uncertainty bounds were calculated for the Gassmann $\mathrm{V}_{p}$ predictions, and it was found that empirical $\mathrm{V}_{p}$ predictions were close to the upper limits of the Gassmann $\mathrm{V}_{p}$ predictions. We therefore concluded that a partial verification of the internal consistency of the empirical equations was demonstrated.
\end{abstract}

\section{INTRODUCTION}

Hamilton (1980) states: "Geoacoustic models of the seafloor are basic to underwater acoustics and to marine geological and geophysical studies. A geoacoustic model is defined as a model of the seafloor with emphasis on measured, extrapolated, and predicted values of those properties important in underwater acoustics and those aspects of geophysics involving sound transmission. In general, a geoacoustic model details the true thickness and properties of sediment and rock layers in the seafloor."

A simple geoacoustic model of the seafloor can be constructed from standard Ocean Drilling Project (ODP) shipboard physical properties measurements of compressional wave velocity $\left(\mathrm{V}_{p}\right)$ and porosity $(\phi)$ that have been corrected to in-situ conditions. This type of model can be applied to such problems as the regional mapping of sediment isochrons through seismic stratigraphical approaches.

Within such a geoacoustic model, it is especially important to correct $\mathrm{V}_{p}$ measurements accurately to in-situ conditions, as $\mathrm{V}_{p}$ is used to convert arrival times to equivalent sediment thickness on seismic records. Inaccurate in-situ determinations of $\mathrm{V}_{p}$ could result in inappropriate depth matching of events on synthetic and field seismograms. Well-resolved depth matching of events will act as an independent external check on the validity of such a geoacoustic model.

A more exacting test of the validity of a geoacoustic model is to verify its internal consistency. This can be attempted through the use of theoretical relationships that link the physical and acoustical properties described in the model. Gassmann's theory (1951) is used to predict $\mathrm{V}_{p}$ from $\mathrm{V}_{s}, \phi$, and other physical properties. These predictions can be compared with experimental $\mathrm{V}_{p}$ measurements. A favorable comparison, within the uncertainties of experiment and theory, will act as a good check on the validity of the geoacoustic model as well as the theory.

\footnotetext{
${ }^{1}$ Duncan, R. A., Backman, J., Peterson, L. C., et al., 1990. Proc. ODP, Sci. Results, 115: College Station, TX (Ocean Drilling Program).

2 School of Ocean Sciences, University College, North Wales (present address: BP Research, Sunbury Research Centre, Chertsey Road, Sunbury on Thames, Middlesex TW16 7NL, U.K.

${ }^{3}$ GEOMAR Reseach Centre, Kiel, Federal Republic of Germany.
}

\section{SAMPLES AND EXPERIMENTAL METHODS}

A number of whole-core samples were brought back from Leg 115 for laboratory geotechnical and acoustical tests. The samples are given in Table 1. Conventional consolidation tests were conducted on these samples, allowing void-ratio vs. effective stress curves to be established for each sample. However, a modified consolidation cell was used (Schultheiss, 1981; Hurley, 1989) that allowed ultrasonic compressional and shear wave velocities to be determined at each effective stress increment. Sample preparation techniques and experimental methods are described by Hurley (1989).

\section{EXPERIMENTAL RESULTS}

\section{Void Ratio vs. Effective Stress}

A summary of the void ratio $(e)$ vs. effective stress $\left(\sigma^{\prime}\right)$ experimental results from consolidation tests conducted on the six carbonate samples from Site 709 are shown in Figure 1 and listed in Table 2. Four interesting observations can be made from these data:

1. The unloading portion of the $e \log \sigma^{\prime}$ curves show negligible void ratio rebound.

2. The preconsolidation stress is not well defined for any of the six curves.

3. The shape of the $e \log \sigma^{\prime}$ curves are of a similar form.

Table 1. Description and location of samples used for laboratory geotechnical and acoustical tests.

\begin{tabular}{lcr}
\hline \multicolumn{1}{c}{$\begin{array}{c}\text { Core, section, } \\
\text { interval }(\mathrm{cm})\end{array}$} & $\begin{array}{c}\text { Sample } \\
\text { code }\end{array}$ & $\begin{array}{c}\text { Depth } \\
\text { (mbsf) }\end{array}$ \\
\hline $115-709 \mathrm{C}-13 \mathrm{H}-5,147$ & CARB 1 & 119.27 \\
$115-709 \mathrm{~B}-7 \mathrm{H}-6,144$ & CARB 2 & 60.96 \\
$115-709 \mathrm{C}-10 \mathrm{H}-5,144$ & CARB 3 & 90.36 \\
$115-709 \mathrm{C}-10 \mathrm{H}-5,147$ & CARB 4 & 90.39 \\
$115-709 \mathrm{C}-3 \mathrm{H}-5,146$ & CARB 5 & 20.87 \\
$115-709 \mathrm{C}-5 \mathrm{H}-5,144$ & CARB 6 & 40.24 \\
\hline
\end{tabular}




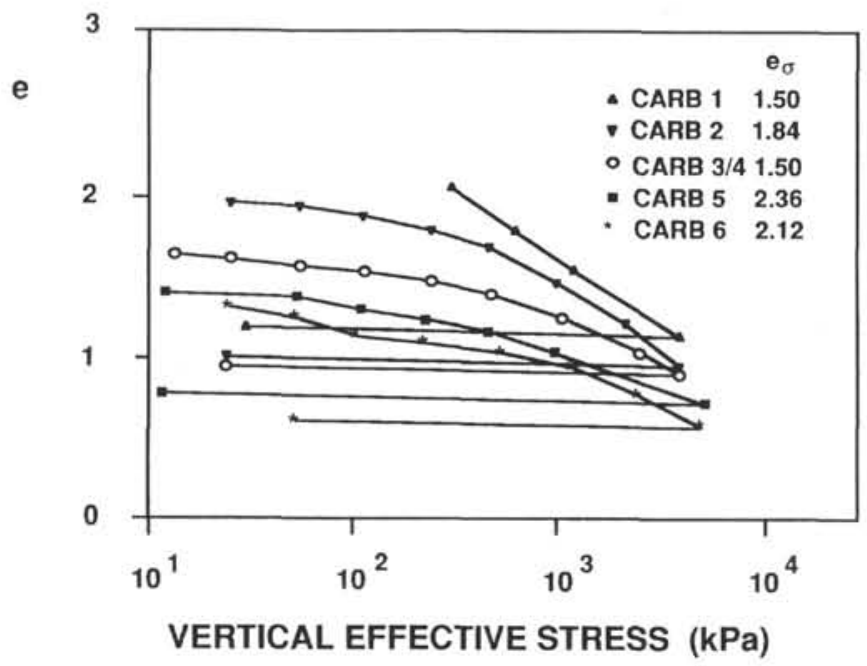

Figure 1. Experimental void ratio vs. vertical effective stress for samples CARB 1 to CARB 6, Site 709.

Table 2. Description and location of samples used for laboratory geotechnical and acoustical tests.

\begin{tabular}{lrccc}
\hline $\begin{array}{c}\text { Sample } \\
\text { code }\end{array}$ & $\begin{array}{c}\sigma \\
(\mathrm{kPa})\end{array}$ & $\begin{array}{c}\mathrm{V}_{s} \\
(\mathrm{~m} / \mathrm{s})\end{array}$ & $\begin{array}{c}\mathrm{V}_{p} \\
(\mathrm{~m} / \mathrm{s})\end{array}$ & $e$ \\
\hline CARB 1 & 13 & - & 1541 & - \\
CARB 1 & 50 & 151 & 1539 & 1.37 \\
CARB 1 & 100 & 167 & 1542 & 1.30 \\
CARB 1 & 200 & 183 & 1549 & 1.25 \\
CARB 1 & 450 & 217 & 1566 & 1.18 \\
CARB 1 & 1000 & 277 & - & 1.07 \\
CARB 2 & 12 & 130 & 1527 & 1.80 \\
CARB 2 & 25 & 140 & 1530 & 1.78 \\
CARB 2 & 50 & 142 & 1533 & 1.76 \\
CARB 2 & 100 & - & 1539 & - \\
CARB 2 & 400 & 190 & 1551 & 1.57 \\
CARB 3 & 12 & 135 & 1536 & 1.57 \\
CARB 3 & 100 & 154 & 1544 & 1.48 \\
CARB 3 & 200 & 202 & 1561 & 1.42 \\
CARB 3 & 400 & 245 & 1570 & 1.35 \\
CARB 4 & 12 & 82 & 1524 & 1.57 \\
CARB 4 & 100 & 105 & 1517 & 1.47 \\
CARB 4 & 200 & 125 & 1527 & 1.42 \\
CARB 4 & 400 & 179 & - & 1.35 \\
CARB 4 & 800 & - & 1569 & 1.24 \\
CARB 5 & 12 & 92 & 1520 & 2.35 \\
CARB 5 & 25 & 121 & 1528 & 2.27 \\
CARB 5 & 50 & 141 & 1533 & 220 \\
CARB 5 & 150 & 134 & 1545 & 2.18 \\
CARB 5 & 250 & 162 & - & 2.11 \\
CARB 5 & 400 & - & 1555 & - \\
CARB 6 & 12 & 81 & 1526 & 2.12 \\
CARB 6 & 25 & 87 & 1532 & 2.09 \\
CARB 6 & 50 & 80 & 1540 & 2.07 \\
CARB 6 & 100 & - & 1547 & - \\
CARB 6 & 200 & 185 & - & 1.93 \\
CARB 6 & 400 & 191 & 1566 & 1.80 \\
CARB 6 & 800 & - & 1578 & - \\
\hline & & & &
\end{tabular}

4. For any two samples $a$ and $b$ where $e_{0 a}>e_{0 b}, e_{a}>e_{b}$ at all equivalent effective stresses.

Two important implications can be drawn from these observations:

1. Deformation of the sediments throughout the loading stages of the tests is primarily of the plastic kind. With little elastic deformation during loading, there is negligible apparent elastic void ratio rebound during unloading. This suggests that the void ratio (hence, porosity) measurements for samples from this site do not require significant correction to in-situ stresses. Therefore, the shipboard porosity measurements for Site 709 (Backman, Duncun, et al., 1988) can be taken to represent insitu values. It must be remembered that consolidation tests are carried out on a time scale much shorter than the natural compaction of the sediments in-situ. Therefore, the consolidation tests will not accurately capture the amount of plastic deformation that occurs after a sample has been placed under a stress exceeding what it experienced in-situ. This means, at high effective stresses, the porosities derived from consolidation tests may be slightly greater than those recorded in-situ.

2. As $e_{a}>e_{b}$ at high effective stresses, samples $a$ and $b$ act in a fundamentally different way. This means samples $a$ and $b$ are intrinsically different because of such processes as cementation; therefore, no one sample can be used to obtain a unique relationship between $e$ and $\sigma^{\prime}$ at high effective stresses. However, because the curves are generally of the same form, the following procedure can be applied to obtain a generalized $e$ vs. $\sigma^{\prime}$ relationship: for each sample, the $e\left(\sigma^{\prime}\right)$ values can be normalized by $e_{0}$; the values $e\left(\sigma^{\prime}\right) / \mathrm{e}_{0}$ are vs. $\sigma^{\prime}$ and a best-fit line is put through the results. For the six samples shown in Figure 1, this gives

$$
\phi\left(\sigma^{\prime}\right)=\phi(0)\left(1-[1.4891] 10^{-4} \sigma^{\prime}+[3.6863] 10^{-8} \sigma^{\prime 2}\right),(
$$

with $\phi=e /(1+e)$.

The correlation coefficient for this equation is 0.98 and the standard deviation of the regression is 0.0055 .

\section{$\mathbf{V}_{p}$ and $\mathbf{V}_{s}$ vs. Effective Stress Relationships}

Using a normalization approach similar to that described for porosity, Hurley (1989) obtained the following relationships for the variations of $\mathrm{V}_{p}$ and $\mathrm{V}_{s}$ vs. effective stress:

$$
\begin{aligned}
\mathrm{V}_{p}\left(\sigma^{\prime}\right)= & \mathrm{V}_{p}(0)\left(1+[5.7776] 10^{-5} \sigma^{\prime}-\right. \\
& {\left.[2.177] 10^{-8} \sigma^{\prime}\right) \text { and } }
\end{aligned}
$$

The correlation coefficients and standard deviation of regression for these equations are $0.90,0.82,0.048$, and 0.195 , respectively.

\section{$\mathbf{V}_{p}, \mathbf{V}_{s}$, and $\phi$ vs. Depth Empirical Equations}

It is the variation of $\mathrm{V}_{p}, \mathrm{~V}_{s}$, and $\phi$ with depth, not effective stress, that is usually required. To obtain such relationships, effective stress and temperature corrections can be applied to $\mathrm{V}_{p}$, $\mathrm{V}_{s}$, and $\phi$ measurements collected at ambient laboratory conditions. This task is simplified by a number of factors: $\phi$ rebound is minimal (see "Samples and Experimental Methods" section, this chapter), and no effective stress correction needs be applied to the $\phi$ measurements; $\mathrm{V}_{s}$ and $\phi$ are effectively temperature independent; and $V_{p}$ and $V_{s}$ shipboard measurements on samples from Site 709 show a negligible systematic depth dependence (Backman, Duncan, et al., 1988).

Considering the porosity first, a linear regression equation can be fit through the uncorrected shipboard laboratory data for Hole 709. The following relationship, with depth $(z)$ in meters results:

$$
\phi(z)=0.6735-0.000543 z
$$


Equations for $\mathrm{V}_{p}$ and $\mathrm{V}_{s}$ vs. depth for Hole 709A (uncorrected for temperature) can be obtained by converting effective stress to depth below seafloor in Equations 2 and 3, and then using averaged values of shipboard $\mathrm{V}_{p}$ and $\mathrm{V}_{s}$ measurements. To convert effective stress to depth below seafloor $(z)$, an effective stress profile is required. Hurley (1989) obtained a linear relationship for Site 709A (Eq. 5), and using this with $\mathrm{V}_{p}(0)=1530$ $\mathrm{m} / \mathrm{s}$ and $\mathrm{V}_{s}(0)=110 \mathrm{~m} / \mathrm{s}$ gives

$$
\begin{gathered}
\sigma^{\prime}(\mathrm{kPa})=6.16 z \text { (mbsf), } \\
\mathrm{V}_{p}=1530+0.5447 z-0.00126 z^{2}, \text { and } \\
\mathrm{V}_{s}=110+1.46 z-0.0048 z^{2} .
\end{gathered}
$$

\section{Shear Modulus vs. Depth Relationship}

The shear modulus (G) is simply related to $\mathrm{V}_{s}$ and $\phi$ :

$$
\mathrm{G}=\mathrm{V}_{s}^{2} \rho_{s}+\phi \mathrm{V}_{s}^{2}\left(\rho_{f}-\rho_{s}\right) .
$$

Here, $\rho_{f}$ and $\rho_{s}$ are the fluid and solid moduli, respectively. Given typical values of $\rho_{s}=2720 \mathrm{~kg} / \mathrm{m}^{3}, \rho_{f}=1024 \mathrm{~kg} / \mathrm{m}^{3}$, $\mathrm{V}_{s}(0)=110 \mathrm{~m} / \mathrm{s}$ and $\phi(0)=0.65$. Combining Equations 4,6 , and 8 , it is possible to obtain an unwieldy fifth-degree polynomial empirical equation for the variation of $G$ with depth. A simplified expression can be obtained by fitting a third-degree polynomial to the values calculated using the fifth-degree polynomial:

$$
\mathrm{G}(z)(\mathrm{MPa})=17.5+0.7013 z-0.01564 z^{2} .
$$

It is useful to determine if the variation of $\phi$ with depth (Eq. 4) or the variation of $\mathrm{V}_{s}$ with depth (Eq. 6) dominates Equation 9. To do this, the following procedure can be applied: Equation 8 can be differentiated with respect to $V_{s}$ and $\phi$, holding $\phi$ and $\mathrm{V}_{s}$ constant, respectively, to obtain $\left(\delta \mathrm{G} / \delta \mathrm{V}_{s}\right)_{\phi}$ and $(\delta \mathrm{G} / \delta \phi)_{\mathrm{V}_{s}}$. To a first approximation, the $z^{2}$ term in Equation 6 can be ignored, allowing $\delta \phi / \delta z$ and $\delta \mathrm{V}_{s} / \delta z$ to be simply established. These partial differentials can be combined to give a linear expression for the variation of $\mathrm{G}$ with $z$ (as in Eq. 10), and typical values for $\mathrm{V}_{s}(0)$ and $\phi(0)$ can be substituted in this equation. Hence, an assessment of the roles of $V_{s}(z)$ and $\phi(z)$ can be made.

$$
\begin{aligned}
\mathrm{G}(z)= & \mathrm{G}(0)+\left(\delta \mathrm{G} / \delta \mathrm{V}_{s}\right)_{\phi}\left(\delta \mathrm{V}_{s} / \delta z\right) z \\
& +(\delta \mathrm{G} / \delta \phi)_{\mathrm{V}_{s}}(\delta \phi / \delta z) z .
\end{aligned}
$$

With the procedure outlined above, with $\mathrm{V}_{s}(0)=110 \mathrm{~m} / \mathrm{s}$ and $\phi(0)=0.65$, the results shown in Figure 2 are defined. It is apparent that the third term in Equation 10 is small in comparison with the second term. This means that the change in porosity with depth-caused by effective stress-is virtually unimportant as far as the shear modulus is concerned, and it is the variation of $\mathrm{V}_{s}$ with depth that is the controlling factor.

There are some points worth noting concerning the analysis in this section. Care must be taken when combining empirical polynomial equations, and caution must be used when applying such combinations (e.g., for Eq. 9). Simply dropping higher order terms (as was done for Eq. 6 above when calculating Eq. 10) is inadvisable in most cases. However, attempting to differentiate higher order polynomials (as could have been done when calculating Eq. 10) is a greater crime. The results shown in Figure 2, as calculated from Equation 10, are a first-order approximation, which is clearly inadequate in capturing the full dependence of $\mathrm{G}$ with depth. However, these results are significant because they show that the variation of porosity with depth is not important. This conclusion would be made (but would be more difficult to show) if the higher order terms were retained.

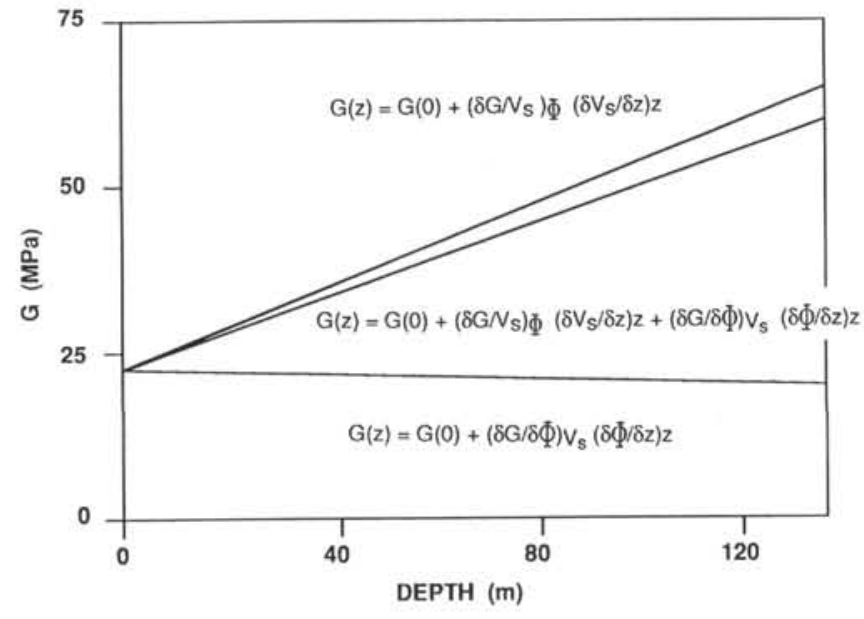

Figure 2. Relative effect of the variation of $\phi$ and $V_{s}$ with depth on calculated shear modulus $(G)$ for carbonate sediments from Site 709.

\section{Internal Consistency of Empirical $\mathbf{V}_{p}, \mathbf{V}_{s}$, and $\phi$ vs. $\sigma^{\prime}$ Equations}

\section{Gassmann Theory}

Gassmann's theory (1951) relates $V_{p}$ to measurable physical and acoustical properties of sediments. If Gassmann's theory can be successfully applied to a simple geoacoustical model, then verification of the internal consistency of the model will be achieved. The following properties were used in Gassmann's equation: the porosity $(\phi)$, the grain density $\left(\rho_{s}\right)$, the fluid density $\left(\rho_{f}\right)$, the fluid modulus $\left(K_{f}\right)$, the grain modulus $\left(K_{s}\right)$, the frame bulk modulus $\left(\mathrm{K}_{b}\right)$, and the shear modulus $(\mathrm{G})$. These physical properties can be used to define the bulk constrained modulus (M) and bulk density $(\rho)$, and hence $\mathrm{V}_{p}$.

$$
\begin{gathered}
\mathrm{M}=\mathrm{K}_{b}+4 \mathrm{G} / 3+\left(1-\mathrm{K}_{b} / \mathrm{K}_{s}\right)^{2} / \\
{\left[\phi / \mathrm{K}_{f}+(1-\phi) / \mathrm{K}_{s}-\left(\mathrm{K}_{b} / \mathrm{K}_{s}^{2}\right)\right],} \\
\rho=\phi \rho_{f}+(1-\phi) \rho_{s}, \text { and } \\
\mathrm{V}_{p}=(\mathrm{M} / \rho)^{1 / 2} .
\end{gathered}
$$

\section{Uncertainty in $\mathbf{V}_{p}$ Predictions from Gassmann's Theory}

It is often assumed that theoretical predictions are accurate, whereas experimental measurements have an associated experimental uncertainty. However, in most cases, uncertainties within theoretical predictions will be expected because of uncertainties in the input parameters used in the prediction. If the uncertainty of the input parameters are known, then a guide to the uncertainty of the prediction can be obtained. The following method can be used for determining the approximate total uncertainty in the predictions yielded by Gassmann's equation (combination of Eqs. 10, 11, and 12):

1. Obtain eight expressions for the partial differential of Gassmann's equation with respect to each of the eight input parameters.

2. Substitute the experimental values of the input parameters into each of these eight expressions and square the results.

3. Square the uncertainty for each of the eight input parameters.

4. For each input parameter, obtain the product of Steps 2 and 3 above.

5. Take the square root of the sum of the eight products in Step 4. 
The above procedure yields $\Delta \mathrm{V}_{p}$, the total uncertainty in $\mathrm{V}_{p}$. The significance of the uncertainty of any input parameter on $\Delta \mathrm{V}_{p}$ can be gauged by the magnitude of the normalized uncertainty component $U$ for that parameter. For each of the eight input parameters, $U$ is defined by the value obtained in Step 4 above divided by $\Delta \mathrm{V}_{p}{ }^{2}$. Therefore, the sum of the eight normalized uncertainty components is equal to unity.

\section{Applying Gassmann's Theory to Empirical $\mathbf{V}_{s}$ and $\phi$ vs. $\sigma^{\prime}$ Equations}

Equations 7, 10, 11, and 12 can be used to predict the variation of $\mathrm{V}_{p}$ with depth for carbonate sediments, providing appropriate values of $\mathrm{K}_{f}, \mathrm{~K}_{s}, \phi, \mathrm{G}$, and $\mathrm{K}_{b}$ are known. Temperaturecorrected predictions and temperature-corrected experimental results for the variation of $\mathrm{V}_{p}$ with depth for carbonate sediments of Site 709 are shown in Figure $3\left(\mathrm{~V}_{p}\right.$ values have been multiplied by the ratio $1458 / 1526$ to correct to in-situ temperature conditions). Predictions of $\mathrm{V}_{p}+\Delta \mathrm{V}_{p}$ and $\mathrm{V}_{p}-\Delta \mathrm{V}_{p}$ were made with the input parameters given in Table 3 and the procedure outlined in the section above. The experimental data points, along with their uncertainty bounds, are taken for sample with $\mathrm{V}_{p}(0)=1529 \mathrm{~m} / \mathrm{s}$ and $e_{0}=1.86(\phi=0.65)$. The uncertainty bounds for $\mathrm{G}, \mathrm{K}_{f}, \phi, \rho_{f}$, and $\rho_{s}$ were calculated from experimetnal uncertainties as discussed in Backman, Duncan, et al., 1988). The error bounds for $K_{s}$ and $K_{b}$ were estimated. The importance of this latter assumption is discussed below.

Looking at Figure 3, it can be seen that the $\mathrm{V}_{p}$ increase with depth. This is caused by the decrease of $\phi$ with depth, coupled with the increase of $\mathrm{G}$ and $\mathrm{K}_{b}$ with depth. It is apparent that there is an overlap between the lower uncertainty bound of the

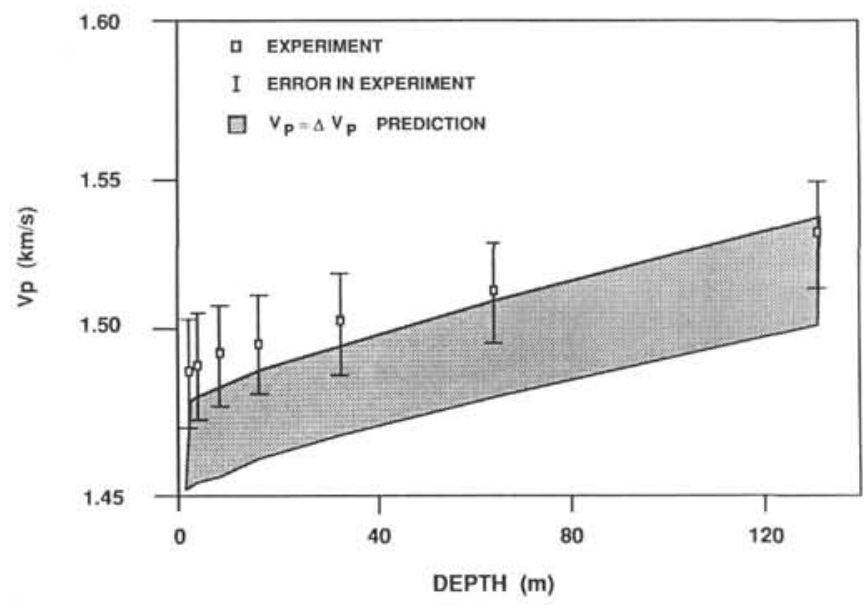

Figure 3. Experimental and predicted $\mathrm{V}_{p}$ vs. depth data for carbonate sediments from Site 709.

Table 3. Input parameters to Gassmann's equation and uncertainty equations.

\footnotetext{
Fluid density $\left(\rho_{f}\right)=1024+10 \mathrm{~kg} / \mathrm{m}^{3}$

Grain density $\left(\rho_{s}\right)=2720+27 \mathrm{~kg} / \mathrm{m}^{3}$

Grain bulk modulus $\left(\mathrm{K}_{s}\right)=(6.3 \pm 1.26) 10^{10} \mathrm{~Pa}$

Fluid bulk modulus $\left(\mathrm{K}_{f}\right)=(2.39+0.024) 10^{9} \mathrm{~Pa}$

Porosity $(\phi)$ given by Eq. 1 with $\phi(0)=0.65$ and an uncertainty of $5 \%$

Shear modulus (G) given by Eqs. 1, 3, and 8 with an uncertainty of $25 \%$

Frame bulk modulus $\left(\mathrm{K}_{b}\right)=2.17 \mathrm{G}$ with an uncertainty of $25 \%$
}

experimental $\mathrm{V}_{p}$ data and the upper uncertainty bound of the $\mathrm{V}_{p}$ predictions. Therefore, a verification of Gassmann's equation, although not totally convincing, could be claimed. It is interesting to note that experimental data are systematically greater than the $\mathrm{V}_{p}$ predictions, and, further, that this difference decreases with increasing depth. Although these observations could be entirely accounted for by the respective uncertainties in experiment and prediction, there is the possibility that the Gassmann theory is lacking and that some additional effective stress (or depth) dependent parameter is also affecting the experimental results.

Biot's (1956) theory has an additional permeability dependent term that can give $\mathrm{V}_{p}$ predictions a few percentage points greater than those given by Gassmann's theory. Gassmann's theory is a low-frequency approximation of Biot's theory, and, at the frequencies at which the measurements were made $(1 \mathrm{MHz})$, we may expect the Biot effect to be present. The greater the permeability, the greater the additional Biot effect. Measurements show that the permeability of these sediments decreases with increasing depth or increasing effective stress. This is possible support of Biot's additional permeability effect. Further discussions are given in Hurley (1989).

Some comments are required on the numerical values given in Table 3. The fluid properties are for a salinity of $35 \mathrm{ppt}$ and for a temperature of $20^{\circ} \mathrm{C}$. The average grain density value was obtained from measuring the dry weight and dry volume of the samples used for consolidation tests. The grain modulus is a typical value for carbonate sediments taken from Ogushwitz (1985). The uncertainty in porosity is a generous $5 \%$ (i.e., $5 \%$ of $\phi$, which equals 0.03 approximately). The uncertainty in the shear modulus is on the order of $25 \%$, assuming an uncertainty in the shear wave velocity of some $10 \%$.

The frame bulk modulus is the most difficult input parameter to which a value can be assigned. Ogushwitz (1985) uses a value of $\mathrm{K}_{b}=2.17 \mathrm{G}$ for modeling acoustic propagation in carbonate sediments. This relationship agrees with measurements of $K_{b}$ and $G$ conducted on unconsolidated sands (Hurley, 1989). Because no measurements of $\mathrm{K}_{b}$ were obtained for these sediments, this relationship was assumed to hold. Therefore, an uncertainty in $\mathrm{K}_{b}$ of $25 \%$ follows from the uncertainty in G. Note, we have attempted to propagate uncertainties through an empirical equation derived from $\mathrm{K}_{b}$ measurements on a somewhat similar type of sediment. This is a pragmatic approach, and the assumption of the relationship between $\mathrm{K}_{b}$ and $\mathrm{G}$ and the assumption of the magnitude of the uncertainty in $\mathrm{K}_{b}$ are obvious weaknesses in the numerical uncertainty analysis presented in this section.

It is interesting to examine the uncertainties that contribute the greatest to $\Delta \mathrm{V}_{p}$, the total uncertainty in $\mathrm{V}_{p}$. The normalized uncertainty components for the parameters given in Table 2 are shown in Figure 4. The magnitude of any one normalized uncertainty component is given by the thickness of the band it occupies in Figure 4. It can be seen that the total normalized uncertainty is dominated by the contribution from $\mathrm{U}_{\phi}$ and $\mathrm{U}_{\mathrm{K}_{f}}$ at all depths. However, the importance of $\mathrm{U}_{\mathrm{K}_{f}}$ decreases with increasing depth as the sediment becomes stiffer. Therefore, the contributions from $\mathrm{U}_{\mathrm{K}_{b}}$ and $\mathrm{U}_{\mathrm{G}}$ become increasingly important with increasing depth. Note that the constraint on the relationship between $\mathrm{K}_{b}$ and $\mathrm{G}$ means that $\mathrm{U}_{\mathrm{K}_{b}}$ is $2.17 \mathrm{U}_{\mathrm{G}}$. The importance of the $\mathrm{U}_{\Delta_{f}}, \mathrm{U}_{\Delta_{s}}$, and $\mathrm{U}_{\mathrm{K}_{s}}$ components are negligible.

\section{CONCLUSIONS}

Empirical relationships for the variation of porosity $(\phi)$ and compressional and shear wave velocity $\left(\mathrm{V}_{p}\right.$ and $\left.\mathrm{V}_{s}\right)$, with effective stress $\left(\sigma^{\prime}\right)$, have been established for carbonate sediments from Site 709. Using a simple $\sigma^{\prime}$ vs. depth ( $z$ ) relationship, nontemperature-corrected empirical equations for $\mathrm{V}_{p}, \mathrm{~V}_{s}$, and $\mathrm{G}$ vs. 


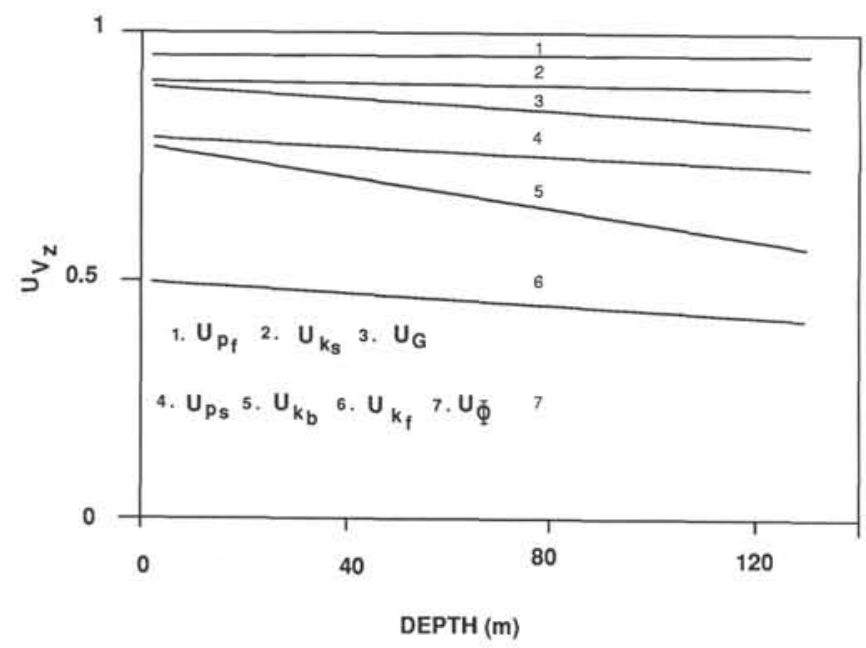

Figure 4. Normalized uncertainty components vs. depth for carbonate sediments from Site 709.

$z$ relationships were obtained. These experimental data were used for various numerical analysis.

Gassmann's equation was used to calculate the variation of $\mathrm{V}_{p}$ with depth. By assigning uncertainties to the parameters used in the prediction, $\mathrm{V}_{p}$ uncertainty bounds were also calculated. It was found that the upper limits of the Gassmann $\mathrm{V}_{p}$ predictions overlap with the lower uncertainty bound of the experimental data. This demonstrates a verification of the internal consistency of the input parameters and Gassmann's equation within experimental and theoretical uncertainty limits.

The variation of $\mathrm{V}_{s}$ with depth was shown to be more important than the variation of $\phi$ with depth when calculating the magnitude of $\mathrm{G}$. At shallow depths, uncertainties in $\phi$ and in $\mathrm{K}_{f}$ outweigh uncertainties in $\mathrm{G}$ or $\mathrm{K}_{b}$ when calculating $\mathrm{V}_{p}$ from
Gassmann's equation. At greater depths, the effect of $\mathrm{G}$ and $\mathrm{K}_{b}$ increase in importance over $\mathrm{K}_{f}$. This implies that $\mathrm{V}_{p}$ predictions are rather insensitive to variations in $\mathrm{G}$ and $\mathrm{K}_{b}$ at shallow depths and are strongly dependent on $\phi$ and $\mathrm{K}_{f}$, whereas at greater depths variations in $\mathrm{G}$ and $\mathrm{K}_{b}$ are of increasing importance.

It is important to note that these conclusions are generally applicable for similar carbonate sediments under similar effective stress conditions. However, other experimental results appearing in the literature will be subject to different magnitudes of uncertainties, as will the magnitudes of the uncertainties of the input parameters to Gassmann's equation. Therefore, these conclusions are not necessarily universally applicable and serve only to account for the experimental and predicted $V_{p}$ data presented within this paper. Their generality can only be verified through the application of similar techniques to a larger data base.

\section{REFERENCES}

Backman, J., Duncan, R. A., et al., 1988. Proc. ODP, Init. Repts., 115: College Station, TX (Ocean Drilling Program).

Biot, M. A., 1956. Theory of propagation of elastic waves in a fluid saturated porous solid, Parts I and II. J. Acoust. Soc. Am., 28:168191.

Gassmann, F., 1951. Uber die elastizität poroser medien. Vierteljahrsschr. Naturforsch. Ges. Zuerich, 96:1-22.

Hamilton, E. L., 1980. Geoacoustic modeling of the seafloor. J. Acoust. Soc. Am., 68:1313-1340.

Hurley, M. T., 1989. Application of Biot's theory to sea-bed sediments [Ph.D thesis]. Univ. Wales.

Ogushwitz, P. R., 1985. Applicability of Biot's theory. III. Wave speeds vs. depths in marine sediments. J. Acoust. Soc. Am., 77:453-464.

Schultheiss, P. J., 1981. Simultaneous measurement of $P$ and $S$ wave velocities during conventional laboratory soil testing procedures. Mar. Geotechnol., 4:343-367.

Date of initial receipt: 11 September 1989

Date of acceptance: 19 January 1990

Ms 115B-191 